\title{
Facing the Emergency Department crisis in Italy
}

\author{
Daniele Coen, ${ }^{1}$ Ivo Casagranda, ${ }^{1}$ Mario Cavazza, ${ }^{1}$ Gianfranco Cervellin, ${ }^{1}$ Lorenzo Ghiadoni, ${ }^{1}$ \\ Roberto Lerza ${ }^{2}$ \\ ${ }^{1}$ Board member of the Emergency Care Journal; ${ }^{2}$ President of the Academy of Emergency Medicine and \\ Care (AcEMC)
}

Since a few years ago health systems in the western countries have a new problem to face: being a Medical Doctor (MD), especially a hospital or a general practice physician, is less and less appealing for the young generations.

Some would say that today's youngsters are not as ready as their parents or grandparents were to tolerate the weight and fatigue inherent in many jobs, but the truth is that being a MD has never been as hard and demanding as it is today. This issue of our journal hosts a letter from a group of young and motivated physicians that raises the question referring to the present situation of Emergency Departments (ED) in Italy. ${ }^{1}$ We apologize to our international readers if we'll try to examine the problem and draft some possible solutions with special reference to our country and its universal health care system, yet we hope that some of our thoughts may prove helpful also in other settings.

\section{Causes}

Looking for the roots of the present situation, two main aspects of the Italian health system need to be considered. On the one hand, the exceedingly high reduction in the number of hospital beds that has taken place since the last years of the former century (from data of the Organization for Security an Co-operation in Europe minus 150.000 or approximately $40 \%$ of total beds) has made Italy one of the countries with the lowest beds/1000 people ratio in Europe. On the other hand, the failure of the chronic care model and its pro-activity, associated with some backwardness and ineffectiveness of general practice, and by and large of the whole of out of hospital medicine, has made it overly difficult for citizens to receive a quick and appropriate answer to their medical needs.

Correspondence: Daniele Coen, Board member of the Emergency Care Journal.

E-mail: dacoen53@gmail.com

Key words: Emergency room; emergency medicine; national health system; health crisis; COVID-19.

Received for publication: 14 December 2021.

Revision received: 14 December 2021.

Accepted for publication: 14 December 2021.

This work is licensed under a Creative Commons Attribution 4.0 License (by-nc 4.0).

(C) Copyright: the Author(s), 2021

Licensee PAGEPress, Italy

Emergency Care Journal 2021; 17:10331

doi:10.4081/ecj.2021.10331
Most General Practitioners (GPs) visit only by appointment (often after many days), don't perform exams in their practices, and rely heavily on hospital specialists. Although things are slowly changing, solo practice is still a widespread framework for family medicine. Moreover, nursing homes and rehab hospitals are insufficient and have long waiting lists, so many patients stay in hospital longer than they should. In this context, citizens are pushed to turn to the ED even for minor ailments or chronic diseases, while patients in need of hospital admission happen to wait days in the ED before they are transferred to a ward. As a consequence, the ED become overcrowded/over boarded ${ }^{2,3}$ and their personnel is overwhelmed. Nurses and doctors are stressed, litigation and burn-out grow. ${ }^{4,5}$ While ED were first career options for young physicians until a few years ago, they are now considered one of the last options and abandoned whenever possible, resulting in the majority of ED being at present heavily understaffed. This happened despite the launch of the specialist school in Italy since 2009/10 for forming MD according to a core curriculum focused for Emergency Medicine. ${ }^{6}$

\section{Looking for solutions}

From what we have briefly summarized, it is clear that there is no quick way out of the present difficult situation and that longlasting work on the whole health system will be necessary before our ED get out of their crisis. Nevertheless, we believe that much could be done in the short and medium term to show the way and to bring the situation under some control. Some possible proposals are as follows.

\section{Short time: out of hospital}

All general practitioners should be forced to open a line of free access to their practice for patients with acute non-severe or chronic conditions. The idea is to offer same-day access to patients that would otherwise turn to the ED. As a first hypothesis, one hour a day should be dedicated to these patients from every doctor in the practice within their or associated studies.

\section{Short time: in hospital}

An immediate and strong mandate should be given to the general manager and chief hospital executives for a quick re-organization of the stream and location of patients within the hospital, in order to reduce crowding and boarding. This should require that admitting departments take direct care of patients waiting in the ED longer than 6-8 hours, in dedicated areas (admission rooms) out of the ED and staffed by personnel from the departments.

ED physicians work should be arranged for no more than two thirds of their time on the Emergency room front line. Hours necessary to complete their requirement should be worked in different settings such as observation units, acute or sub-intensive medical wards, and emergency ambulance services. Although this will not 
immediately respond to the shortage of MD, it could make the job more interesting for the younger generations and more promising in terms of professional growth.

ED physician salary in Italy is one of the lowest in Europe, despite the degree of working and burnout load. Policy for its increase will reduce the escape of MD from EDs and the recruitment of new specialists in the Emergency Medicine school.

Specialization in Emergency Medicine should be assimilated to those of the medical area as regards the access to public competitions for medical positions within and outside the hospital. This will reduce the fear of young MD entering the Emergency Medicine school of having no way out of the ED for the rest of their professional career.

\section{Medium time: out of hospital}

The training of general practitioners should be committed to the University, rather than to regional schools as it is today.

GPs should be required to work in group practices of at least three doctors and one nurse, with the only exception of very isolated areas. At least 6 hours a day of direct access for acute nonsevere conditions should be granted in every individual or aggregate practice.

"Community houses", which are considered as the backbone of the future reform of out of hospital medicine, should not only offer a common roof to the various medical, nursing and social services, but should also become a reference point for training and professional education. As major objectives they should pursue the direct use of technology (ECG, sonography, basic lab) within all medical group practices and the development of common guidelines and protocols to share between the GPs and the specialists to whom they refer.

Pro-active medicine should be endorsed and fragile or potentially deteriorating patients should be followed on a regular basis by their GPs. Home visits by nurses and tele-medicine consultations should be fostered and greatly expanded.

"Community hospitals" of 20/40 beds staffed with nurses and under control of one or more GPs should accept patients discharged from hospital that still need short or medium term assistance and care.

The presence of a physician should be granted $24 / 24 \mathrm{~h}$ in all nursing homes with more than 50 guests, in order to offer the patients adequate medical care while reducing useless and often futile transfers to hospital.

\section{Medium time: in hospital}

Long lasting cuts in the number of hospital beds should be stopped and a reversal initiated. A goal of 4 beds/1000 people should be set as an objective to reach within 5 years.

New formats for the organization and staffing of the ED should be studied, experimented and implemented. It is likely that the same format could not be applied to hospitals of different size and level, yet every effort should be made to preserve the high professional quality that emergency medicine and emergency doctors have reached in the last decades.

Among leading criteria, we propose that physicians working in the ED may have different engagements and career perspectives. It is reasonable to envisage, for instance, specialized emergency physicians acting as long time consultants and younger doctors for other specialist's medical area (e.g. internal medicine) working for a period of 1 to 5 years in the ED before moving to different specialist units. In five years, the latter could close the gap to having an adequate number of specialists in Emergency Medicine.

In a hub and spoke hospital network, different strategies could be used at the different levels. A higher proportion of emergency physicians and residents in emergency medicine (up to $100 \%$ ) could staff the EDs of hubs and teaching hospitals, while a higher number of rotating physicians could be necessary in smaller hospitals where the paucity of doctors is paramount.

In order to develop many of the points that we have dwelled upon, regulatory, administrative and organizational changes are needed and resistances should be envisaged. Yet, introducing more flexibility in the system is the key to its reformation. Out of hospital and hospital medicine should find ways for greater cooperation and integration. Doctors should be allowed to spend part of their professional life within the hospital and part outside. Emergency physicians should be helped particularly in the very next few years to face the great challenges of their job in a supporting environment and the young Authors of the letter that we publish should be reassured in their belief that "Emergency medicine is the most interesting 15 minutes of every other specialty".

They sincerely wish that some of the suggestions here presented will be considered, and help offer the next generation of emergency physicians the future they hope for and deserve.

\section{References}

1. Poggiali E, Barbieri G, Salvatore V, Salinaro F. The Emergency Rooms and Emergency Medicine should not be the answer to the weakness of the Italian National Health System. Emerg Care J 2021;17:10304.

2. Campagna S, Conti A, Dimonte V, et al. Trends and characteristics of emergency medical services in Italy: A 5-years population-based registry analysis. Healthcare (Basel) 2020;8:551.

3. Pines JM, Hilton JA, Weber EJ, et al. International perspectives on emergency department crowding. Acad Emerg Med 2011;18:1358-70.

4. Lin M, Battaglioli N, Melamed M, et al. High prevalence of burnout among US emergency medicine residents: Results from the 2017 national emergency medicine wellness survey. Ann Emerg Med 2019;74:682-90.

5. Verougstraete D, Hachimi Idrissi S. The impact of burn-out on emergency physicians and emergency medicine residents: a systematic review. Acta Clin Belg 2020;75:57-79.

6. Härtel C, Prosen G, Brown R, Dryver E. European core curriculum for emergency medicine, Version 2.0. European Society for Emergency Medicine, 2019. Available from: https://eusem.org/images/Curriculum 2.0 WEB.pdf 